\title{
A New High Speed, High Resolution 2k x 2k CCD Camera for Transmission Electron Microscopes
}

\author{
Y. Jia*, B. Mollon*, P. Mooney*, M. Pan*, B. McGinn*, M. Azimi*, N. Cabilan*, A.J. \\ Gubbens*, M. Lent* \\ *Gatan, Inc., 5794 W. Las Positas Blvd., Pleasanton CA 94588
}

In response to the need for a camera with higher speed and lower noise for both life science and materials science applications, Gatan, Inc. has recently developed the US1000XP CCD camera. This $2 \mathrm{k}$ x 2k CCD camera is a complete redesign of Gatan's US1000 CCD camera combining Gatan's patented multi-speed digital correlated double sampling from the Multi-Scan ${ }^{\mathrm{TM}}$ line of cameras with the multi-port UltraScan line and adding new developments in scintillator technology. 4 readout ports operating at $10 \mathrm{MHz}$ now deliver 0.25 second low-noise full-frame readout and live preview mode. A $1 \mathrm{MHz}$ readout allows ultra-low read noise for low-dose applications. Flexible binning allows readout over a range of image resolutions from $2 \mathrm{x}-8 \mathrm{x}$ yielding effective pixel size/resolution combinations of $28 \mu \mathrm{m} / 1 \mathrm{k} \times 1 \mathrm{k}$ to $112 \mu \mathrm{m} / 256 \times 256$ as well as extreme low-dose previewing up to 30 frames per second at $512 \times 512$.

The camera is built using the same US1000 CCD (2048 x 2048 pixel, $14 \mu \mathrm{m}$ pixel, 100\% fill factor) coupled to a newly developed range of high resolution scintillators in order to improve system resolution and DQE. The new scintillators demonstrate a better tradeoff between thickness and brightness and allow tuning for a variety of purposes. Three phosphor optimizations have been done and are referred to as $\mathrm{P}+, \mathrm{U}+$ and $\mathrm{T}+$. The high resolution $\mathrm{P}+$ scintillator is optimized for higher MTF and its lower sensitivity also allows accumulation of more primary beam thereby reducing shot noise. The $\mathrm{P}+$ is capable of delivering the highest SNR of any of these scintillators when dose is not a consideration. A high resolution carbon graphite sample on a $\mathrm{P}+$ scintillator with FFT inset shows contrast going out to 0.92 Nyquist as shown in figure 1a. The high sensitivity U+ scintillator offers the highest DQE for applications requiring the highest signal to noise ratio under low-dose conditions $\left(10-30 \mathrm{e}^{-} / \mathrm{pixel}\right)$ and is especially well suited for single particle structural biology. The ultra-sensitive $\mathrm{T}+$ scintillator provides an extremely low noise floor and boosts DQE at low spatial frequency for applications such as cryo-tomography and low-dose electron diffraction. When coupled with the up to 30 frames per second readout at $512 \times 512$, the $\mathrm{T}+$ scintillator provides the sensitivity needed to address the low dose conditions created by high-speed readout in in situ studies. A gold and graphite sample image extracted from a 24 frames per second movie on a $\mathrm{T}+$ scintillator is shown in figure $1 \mathrm{~b}$. MTF and DQE comparisons are shown in figures 2 for both $100 \mathrm{kV}$ and 200 $\mathrm{kV}$. The P+ improvements in the US1000XP over the US1000 P scintillator show a significant increase in MTF. U+ improvements in the US1000XP over the original US1000 U scintillator show an increase in DQE.

The new state-of-the-art controller (which replaces the First Light controller) allows reduced readout noise ( $<15 \mathrm{CCD}$ electrons) in $1 \mathrm{MHz}$ readout mode while using Gatan's patented digital correlated double sampling to allow the fast (10 MHz x 4 outputs) readout for setup modes and for high-throughput applications. This camera also has a new mechanical interface which is compatible with the existing UltraScan housing, making it possible for existing UltraScan users 
to upgrade to the new US1000XP camera without sacrificing compatibility with an existing installed energy filter mounted below the camera housing.

The well-balanced integration of fine sampling, enhanced optics and robust scintillator with lownoise and high-speed readout options provides an ideal solution for a broad range of EM applications in biology, materials science and in situ experimentation.

\section{References}

[1] P. Mooney, Methods Cell Biol. 79 (2007) 661

[2] P. Mooney, Microsc. Microanal. 15 (Suppl. 2) (2009) 234

[3] E. Samei et al., Med phys. 25 (1998) 102.

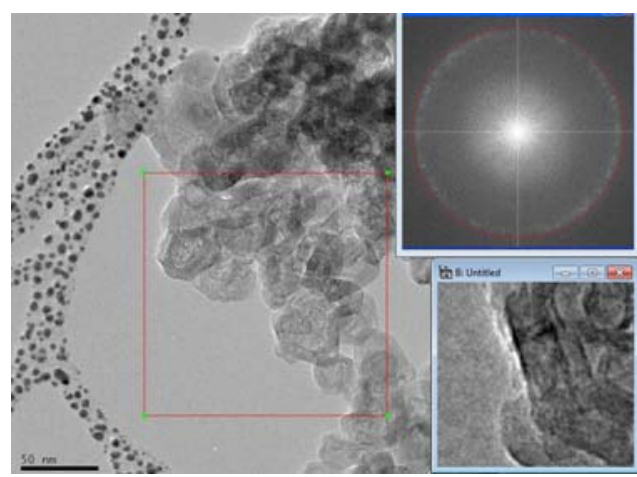

a)

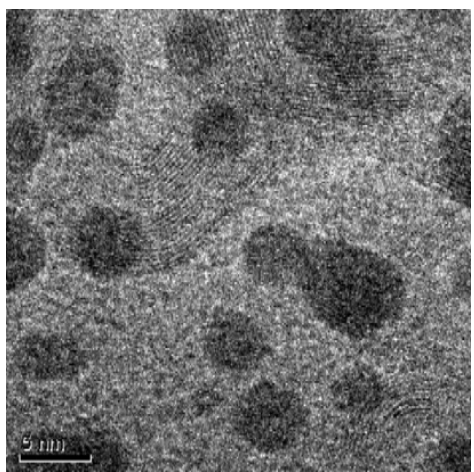

b)

FIG. 1. a) P+ scintillator: Carbon graphite sample, $2 \mathrm{k} x 2 \mathrm{k}$ image, $71 \mathrm{k}$ magnification, $200 \mathrm{kV}$ on FEI Tecnai 20; b) T+ scintillator: Gold and graphite sample, 512 x 512 image extracted from a movie clip at $285 \mathrm{k}$ magnification, 24 frames per second readout, $8 \mathrm{~ms}$ exposure time, $200 \mathrm{kV}$ on FEI Tecnai 20.

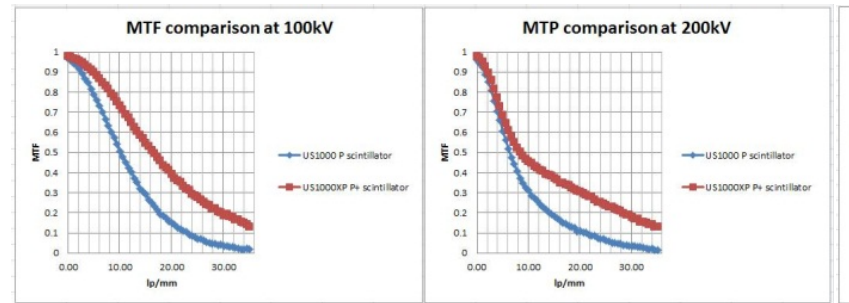

a)

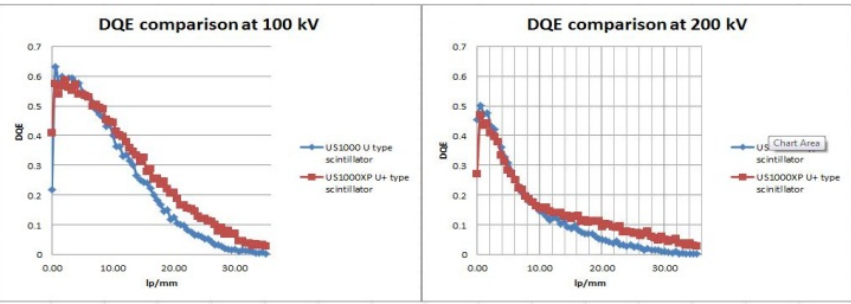

b)

FIG. 2. a) MTF comparison at $100 \mathrm{kV} / 200 \mathrm{kV}$, US1000XP P+ vs. US1000 P with methods outlined in [1] and [2], based on ISO 12233 as described in [3], using a tilted polished edge mounted in the $35 \mathrm{~mm}$ port of the TEM, pairs of uniform illumination images to obtain noise power spectra and a gain measurement using a Faraday cup; b) DQE comparison at $100 \mathrm{kV} / 200$ kV, US1000XP U+ vs. US1000 U. 